\title{
Programming Drug Delivery Kinetics for Active Burst Release with DNA Toehold Switches
}

Mingshu Xiao ${ }^{1}$, Wei Lai ${ }^{1}$, Fei Wang ${ }^{2}$, $\operatorname{Li~Li}^{1}$, Chunhai Fan² and Hao Pei ${ }^{1, *}$

${ }^{1}$ Shanghai Key Laboratory of Green Chemistry and Chemical Processes, School of Chemistry and Molecular Engineering, East China Normal University, 500 Dongchuan Road, Shanghai, 200241, P. R. China

${ }^{2}$ School of Chemistry and Chemical Engineering, and Institute of Molecular Medicine, Renji Hospital, School of Medicine, Shanghai Jiao Tong University, Shanghai 200240, China

*Corresponding author. Email: peihao@chem.ecnu.edu.cn 
The materials include content:

Supplementary Fig. 1 Representative time-lapse TIRF microscopy images of SNAgel and their corresponding fluorescence intensity.

Supplementary Fig. 2 Schematic illustration, TEM images and DLS of AuNP templates, ATP-responsive SNAgel, and ATP-responsive SNAgel in the presence of ATP.

Supplementary Fig. 3 TEM images of SNAgel under different reaction time of BP.

Supplementary Fig. 4 The diameter and the gel thickness of SNAgel under different reaction time of $\mathrm{BP}$.

Supplementary Fig. 5 (a) The fluorescence spectra of the Dox solution $(2 \mu \mathrm{M})$ with increasing molar of SNAgel after incubation in PBS ( $\mathrm{pH}=7.4)$. (b) The fluorescence spectra of SNAgel/Dox $(2 \mu \mathrm{M})$ at the molar of SNAgel as $6 \mathrm{nM}$ in the presence of different concentrations of ATP after incubation.

Supplementary Fig. 6 (a) Fluorescence recovery ratios of SNAgel/Dox in the presence of different concentrations of ATP, GTP, CTP and UTP $(0.2,0.5,1,2,4,8 \mathrm{mM})$. (b) Fluorescence recovery ratios of SNAgel/Dox and cSNAgel/Dox in the presence of 8 mM ATP.

Supplementary Fig. 7 The Zeta potential of SNAgel, and SNAgel/Dox in the absence and presence of ATP.

Supplementary Fig. 8 The hydrodynamic size of SNAgel, SNAgel/Dox, and SNAgel/Dox treated with $4 \mathrm{mM}$ ATP for $5 \mathrm{~min}$.

Supplementary Fig. 9 The release rate of SNAgel/Dox (PBS), cSNAgel/Dox (4 mM ATP), SNAgel/Dox (4 mM ATP), and SNAgel/Dox (PBS+ATP).

Supplementary Fig. 10 The cytotoxicity of SNAgel/Dox with different length of toehold on HeLa cells for different time (1 to $16 \mathrm{~h}$ ).

Supplementary Fig. 11 Flow cytometry measurement of cellular Dox fluorescence intensities and their mean fluorescence intensities in (a) HeLa and (b) HEK 293 cells.

Supplementary Fig. 12 In vitro cytotoxicity of cSNAgel/Dox, SNAgel/Dox and free Dox on HeLa and HEK 293cells for $18 \mathrm{~h}$.

Supplementary Fig. 13 The apoptosis content of HeLa cell induced by Dox, cSNAgel/Dox and SNAgel/Dox for $16 \mathrm{~h}$ using the Annexin V-FITC/PI staining.

Supplementary Table 1. Sequences of DNA used in the experiments

Supplementary Table 2. The diameter of nanoparticles

Supplementary Table 3. The hydrodynamic diameter of nanoparticles 

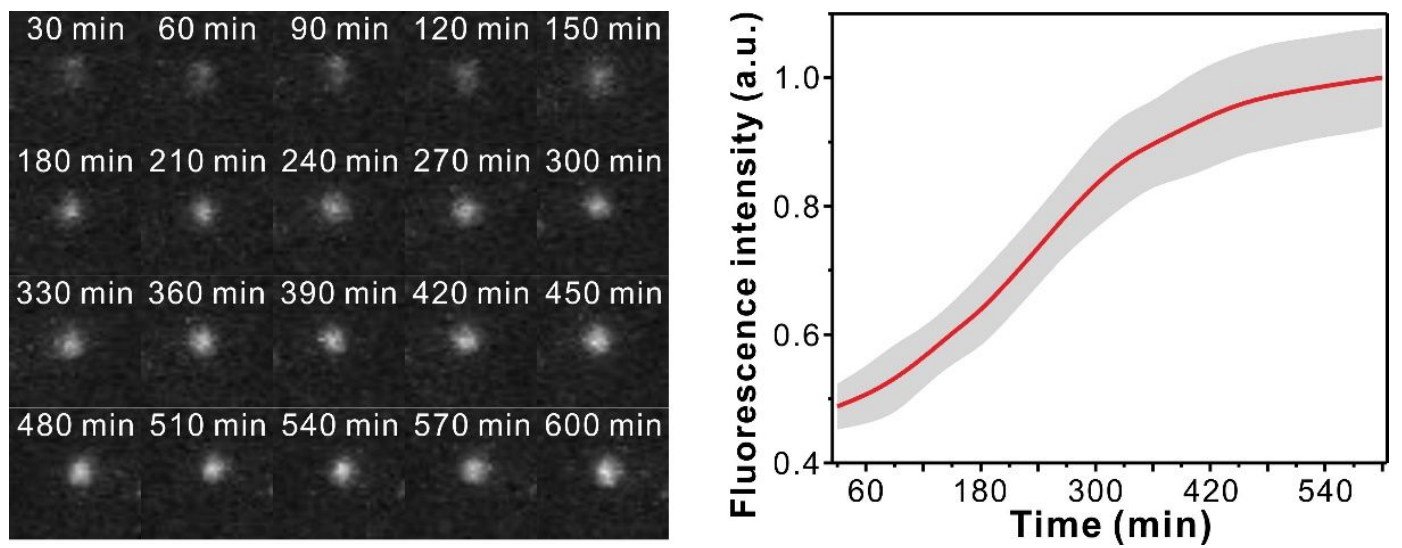

Supplementary Fig. 1 Representative time-lapse TIRF microscopy images of SNAgel and their corresponding fluorescence intensity. Error bars indicate s.d. $(n=3)$.

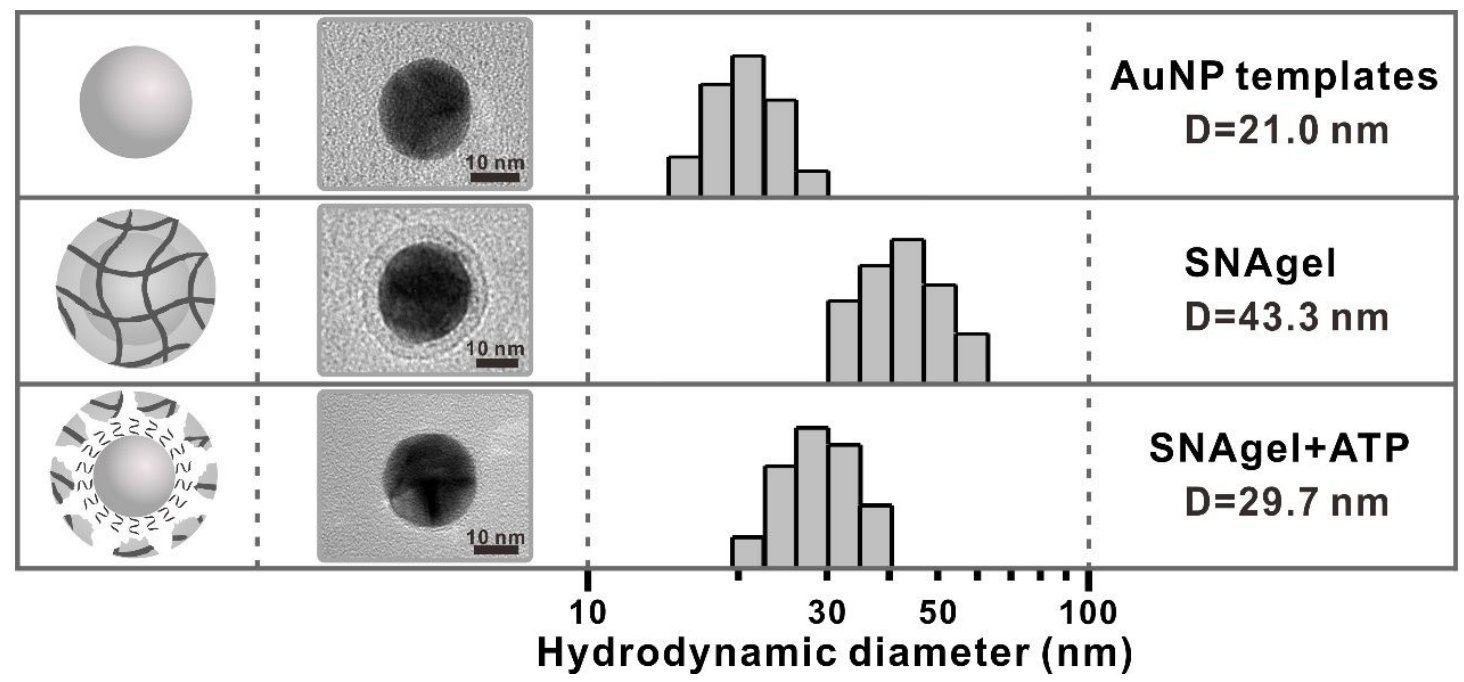

Supplementary Fig. 2 Schematic illustration, TEM images and DLS of AuNP templates, ATP-responsive SNAgel, and ATP-responsive SNAgel in the presence of ATP.

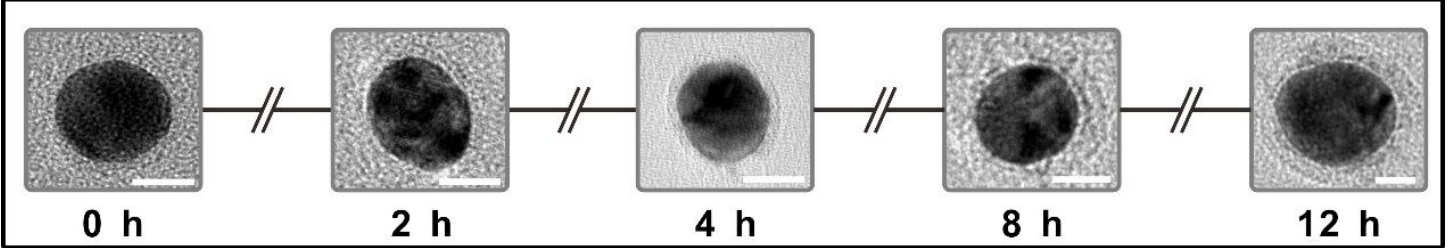

Supplementary Fig. 3 TEM images of SNAgel under different reaction time of BP. Scale bar: $10 \mathrm{~nm}$. 

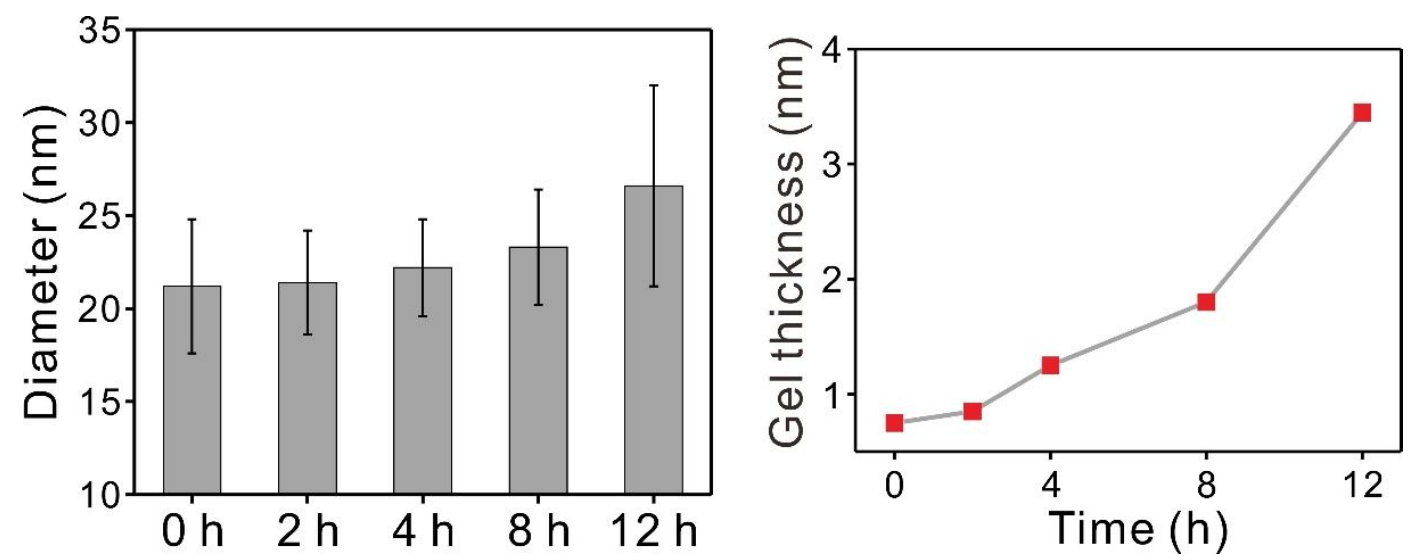

Supplementary Fig. 4 The diameter and the gel thickness of SNAgel under different reaction time of $\mathrm{BP}$.
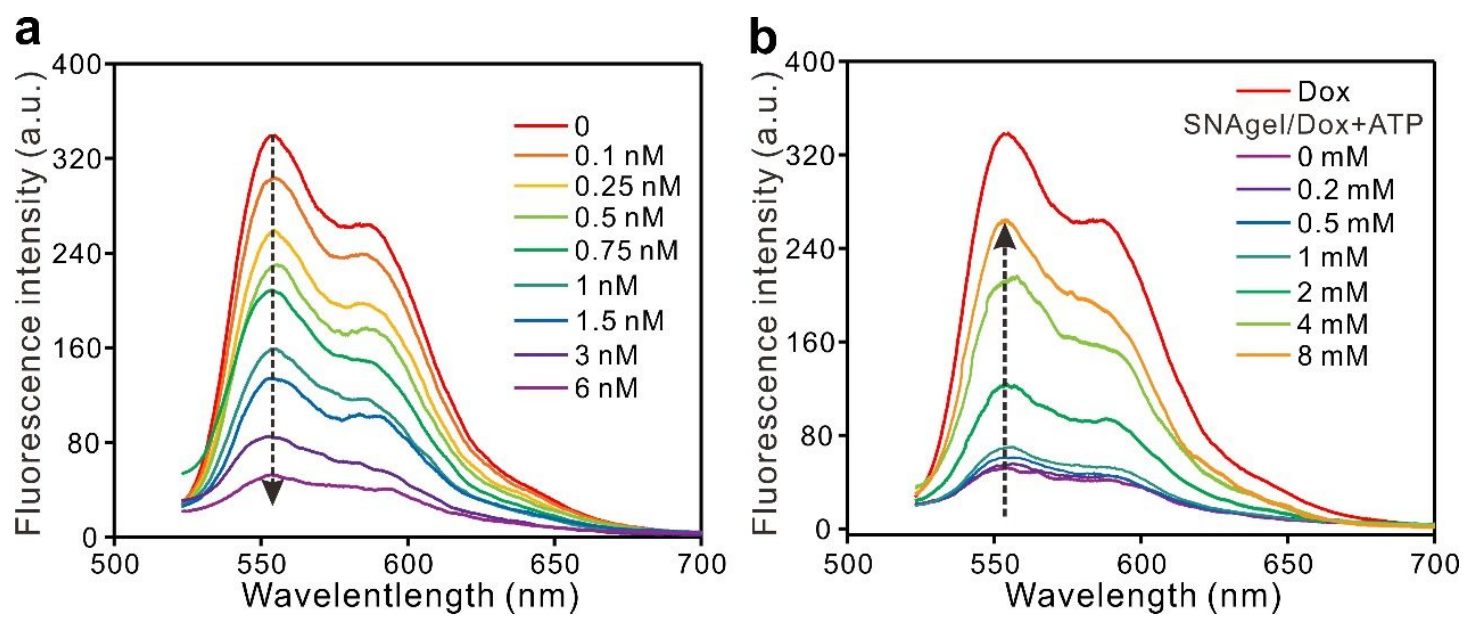

Supplementary Fig. 5 (a) The fluorescence spectra of the Dox solution $(2 \mu \mathrm{M})$ with increasing molar of SNAgel after incubation in PBS $(\mathrm{pH}=7.4)$. (b) The fluorescence spectra of SNAgel/Dox $(2 \mu \mathrm{M})$ at the molar of SNAgel as $6 \mathrm{nM}$ in the presence of different concentrations of ATP after incubation.
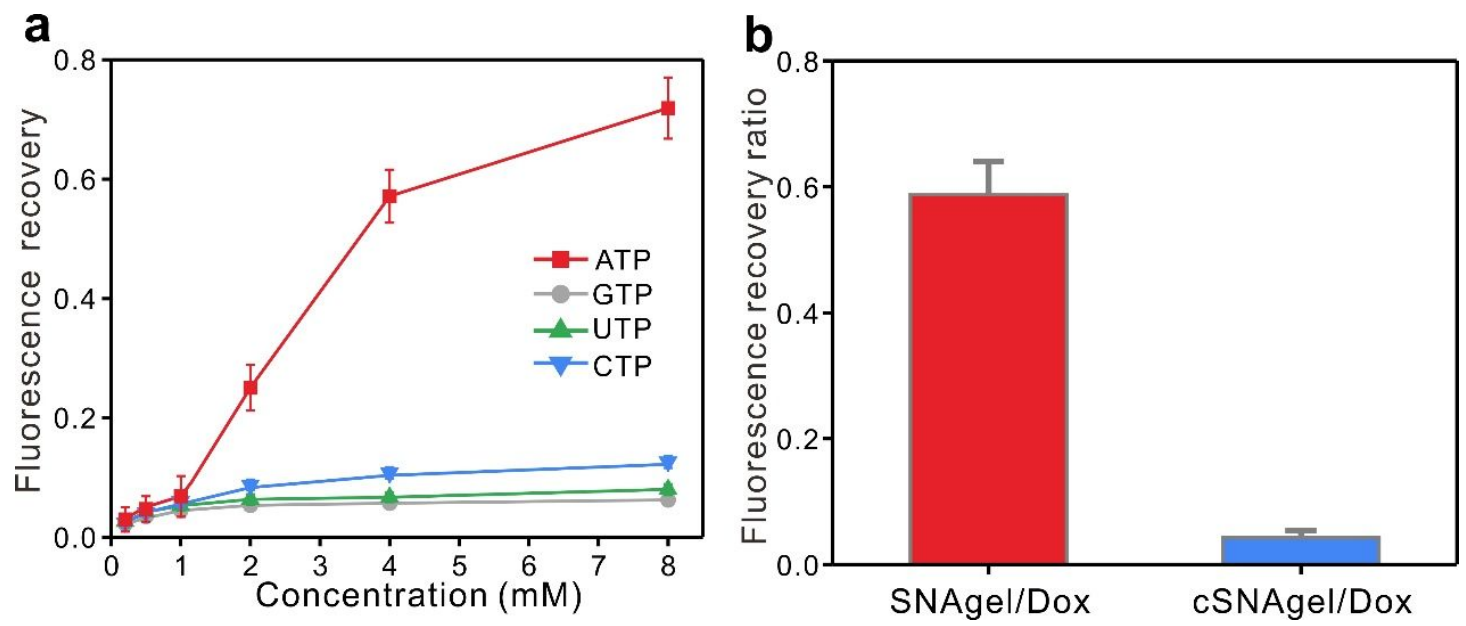

Supplementary Fig. 6 (a) Fluorescence recovery ratios of SNAgel/Dox in the presence 
of different concentrations of ATP, GTP, CTP and UTP $(0.2,0.5,1,2,4,8 \mathrm{mM})$. Error bars indicate s.d. $(n=3)$. CTP, cytidine triphosphate; GTP, guanosine triphosphate; UTP, uridine triphosphate. (b) Fluorescence recovery ratios of SNAgel/Dox and cSNAgel/Dox in the presence of $8 \mathrm{mM}$ ATP. Error bars indicate s.d. $(\mathrm{n}=3)$. The fluorescence recovery ratio is indicated as $\left(\mathrm{F}_{\mathrm{NTP}}-\mathrm{F}\right) /\left(\mathrm{F}_{0}-\mathrm{F}\right)$, where $\mathrm{F}_{0}$ is the fluorescence intensity of Dox in the Dox solution without SNAgel, and $F_{N T P}$ and $F$ are the fluorescence intensities of Dox in SNAgel/Dox at the same Dox concentration with the Dox solution in the presence and absence of NTP, including ATP, CTP, GTP and UTP, respectively.

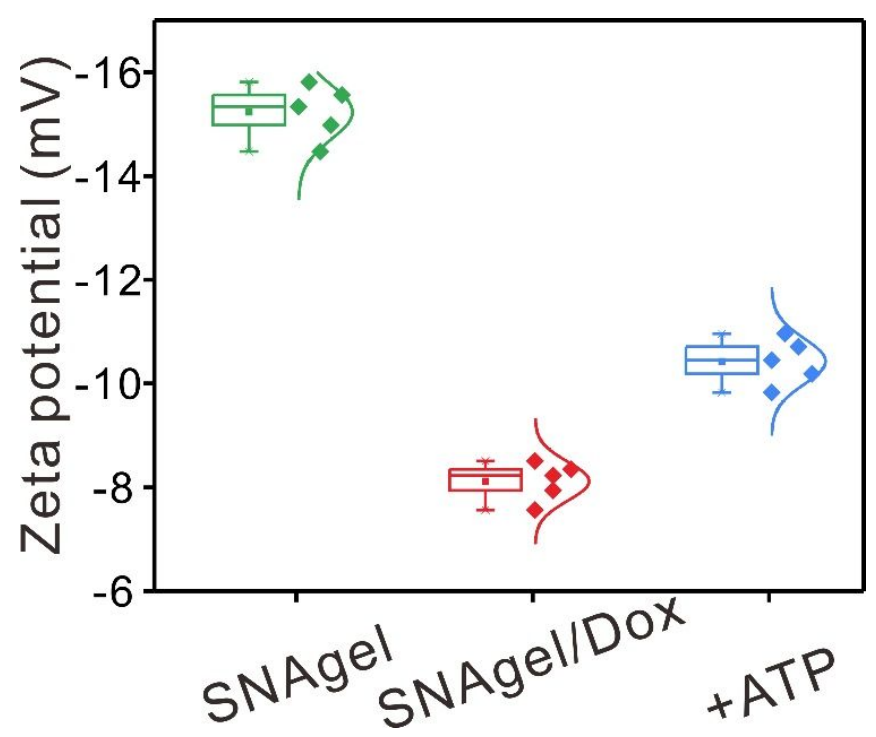

Supplementary Fig. 7 The Zeta potential of SNAgel, and SNAgel/Dox in the absence and presence of ATP.

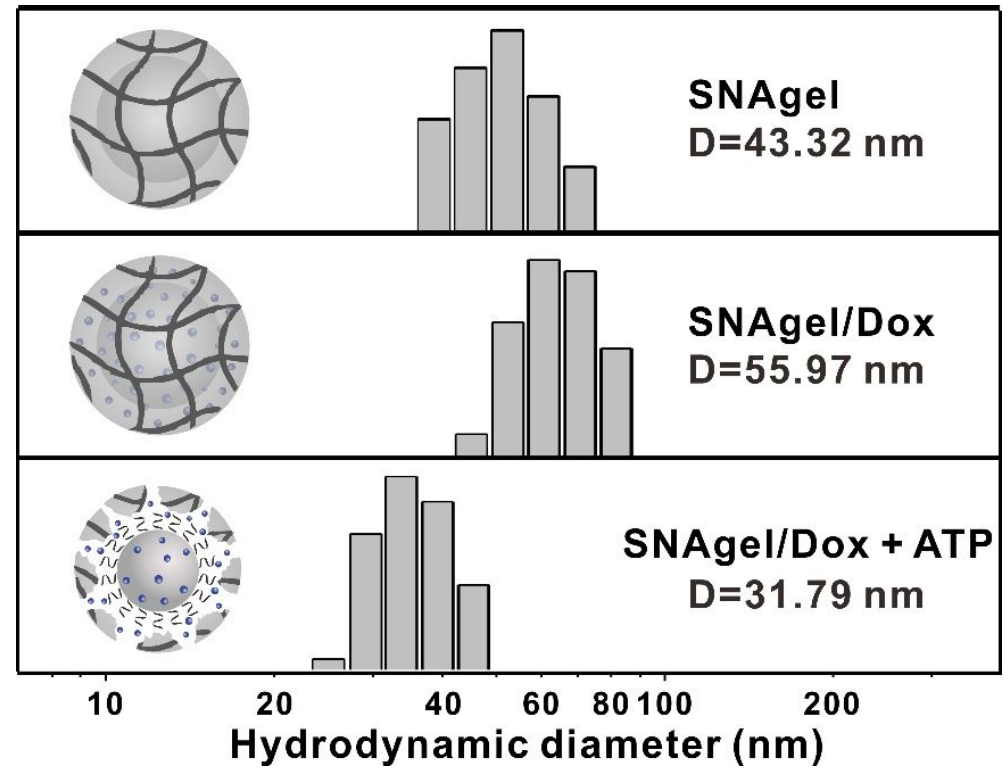

Supplementary Fig. 8 The hydrodynamic size of SNAgel, SNAgel/Dox, and SNAgel/Dox treated with $4 \mathrm{mM}$ ATP for $5 \mathrm{~min}$. 


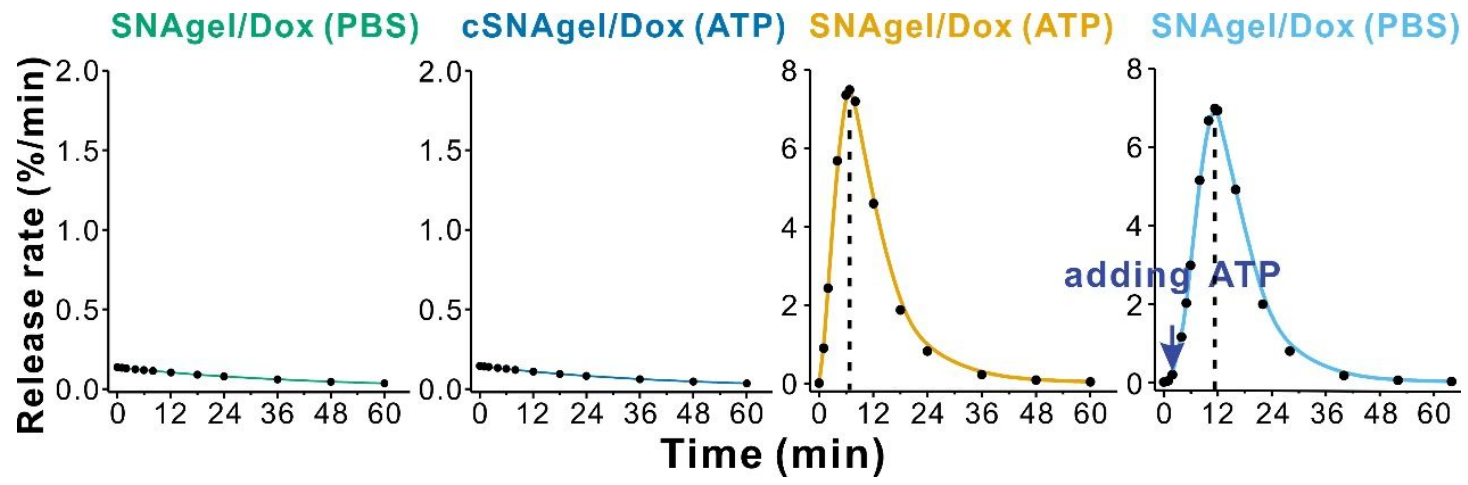

Supplementary Fig. 9 The release rate of SNAgel/Dox (PBS), cSNAgel/Dox (4 mM ATP), SNAgel/Dox (4 mM ATP), and SNAgel/Dox (PBS+ATP).

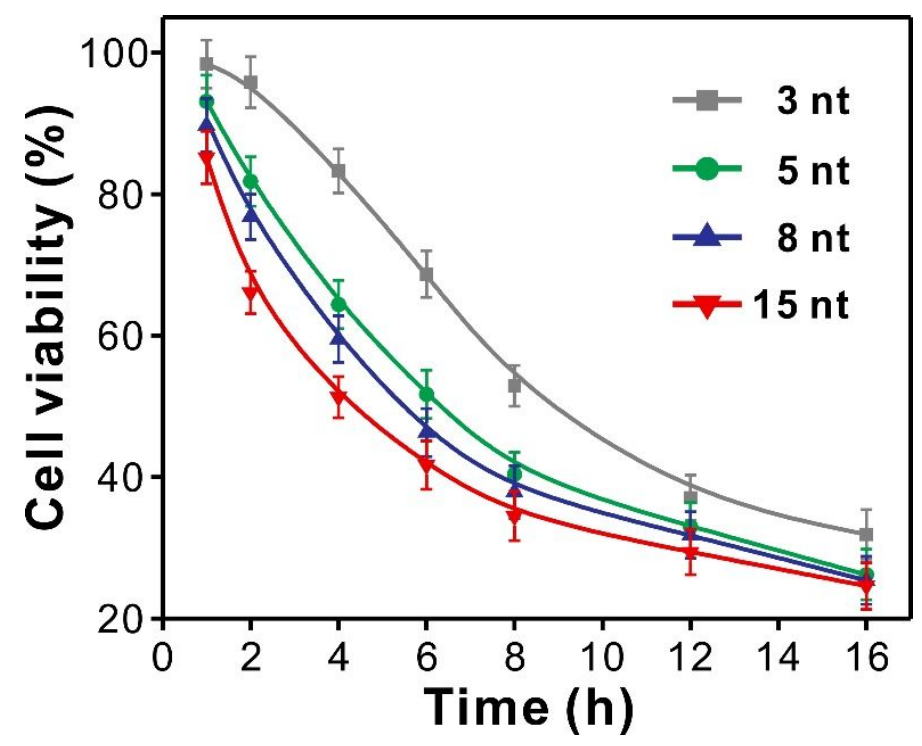

Supplementary Fig. 10 The cytotoxicity of SNAgel/Dox with different length of toehold on HeLa cells for different time (1 to $16 \mathrm{~h}$ ). 

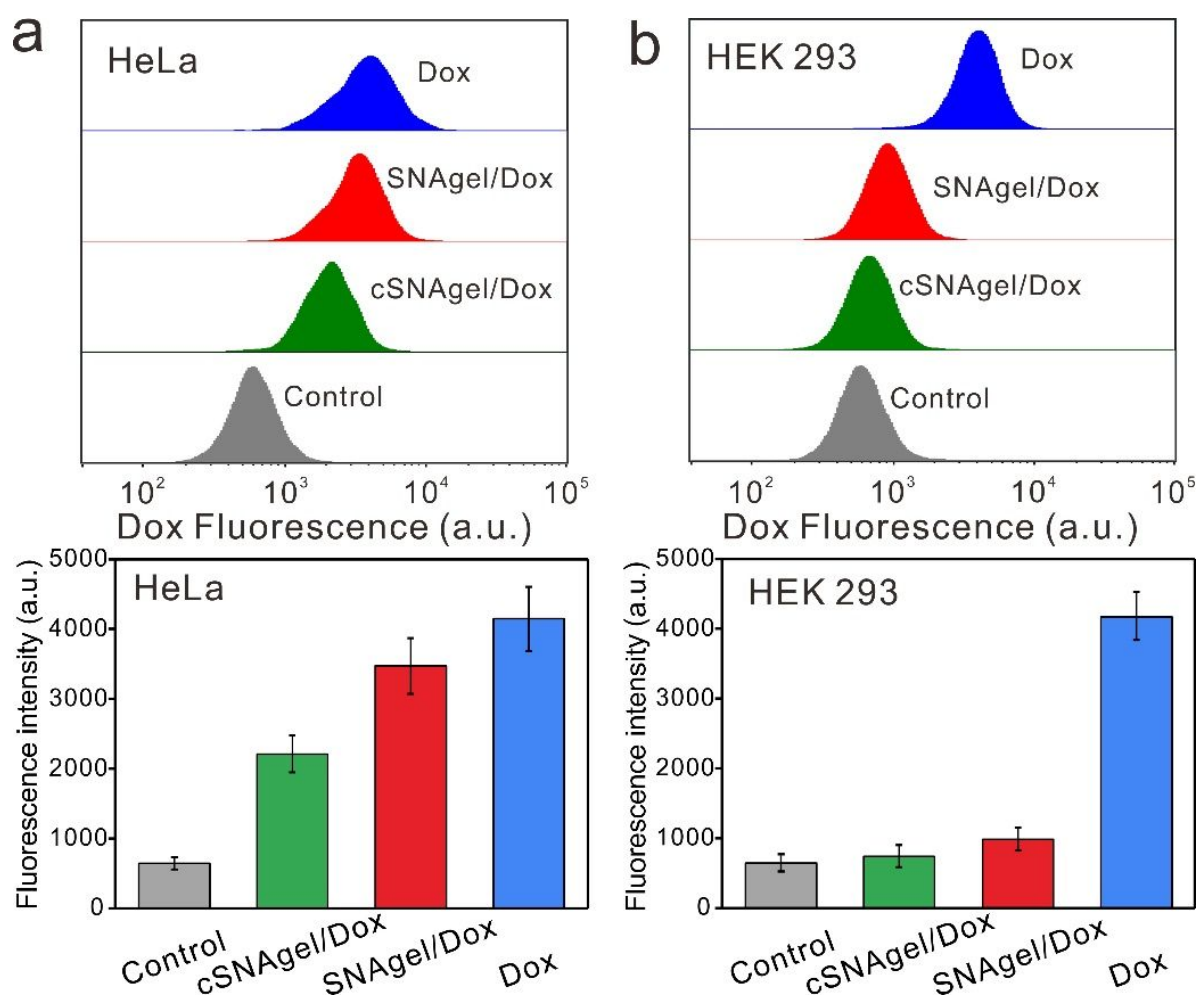

Supplementary Fig. 11 Flow cytometry measurement of cellular Dox fluorescence intensities and their mean fluorescence intensities in (a) HeLa and (b) HEK 293 cells.

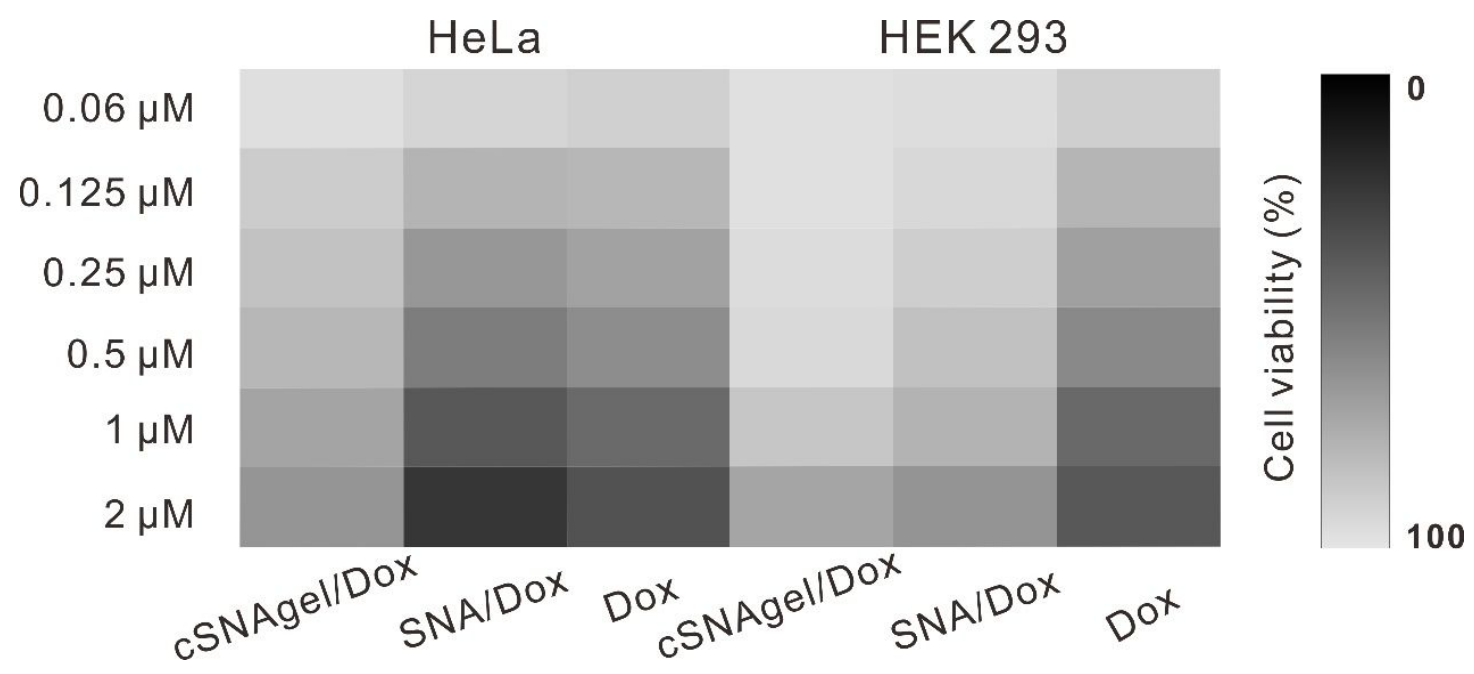

Supplementary Fig. 12 In vitro cytotoxicity of cSNAgel/Dox, SNAgel/Dox and free Dox on HeLa and HEK 293cells for $18 \mathrm{~h}$. 


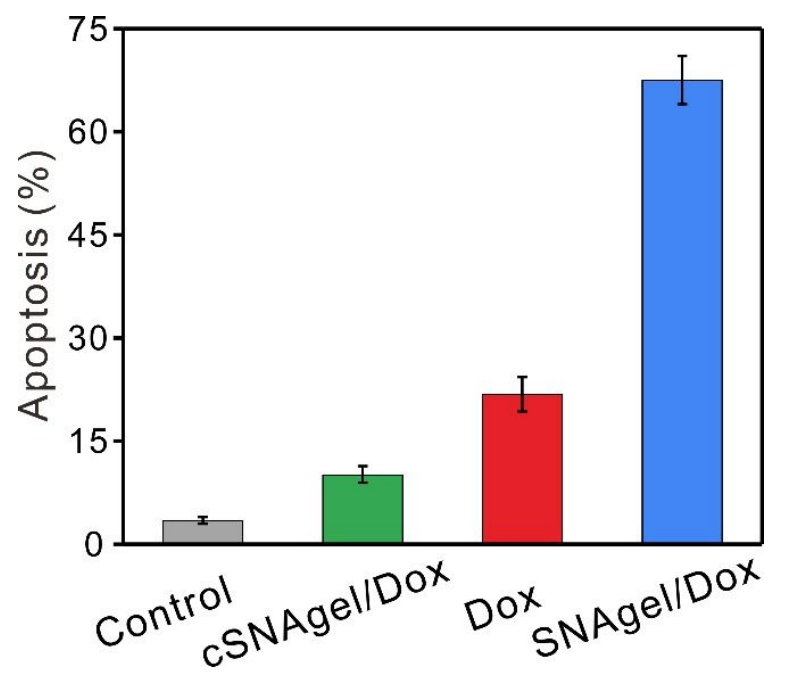

Supplementary Fig. 13 The apoptosis content of HeLa cell induced by Dox, cSNAgel/Dox and SNAgel/Dox for $16 \mathrm{~h}$ using the Annexin V-FITC/PI staining. 
Supplementary Table 1. Sequences of DNA used in the experiments

\begin{tabular}{|c|c|}
\hline Name & Sequence (5' to 3') \\
\hline I & CACCTGGGGGAGTATTGCTAAAGGAAAAA 3'SH C6 \\
\hline $\mathrm{M}_{1} \mathrm{Apt}_{1}$ & $\begin{array}{l}\text { CCTTTAGCAATACTCCCCCAGGTGCCAAATCACCTGGGGGAGTATTGCGG } \\
\text { AGGAAGGT }\end{array}$ \\
\hline $\mathrm{M}_{2} \mathrm{~B}$ & $\begin{array}{l}\text { CACCTGGGGGAGTATTGCTTCAACCTTCACACCTGGGGGAGTATTGCTAA } \\
\text { AGGGCAATACTCCCCCAGGTGATTTGG }\end{array}$ \\
\hline Cy5.5-labeled $\mathrm{M}_{2} \mathrm{~B}$ & $\begin{array}{l}\text { 5'-Cy5.5- } \\
\text { CACCTGGGGGAGTATTGCTTCAACCTTCACACCTGGGGGAGTATTGCTAA } \\
\text { AGGGCAATACTCCCCCAGGTGATTTGG }\end{array}$ \\
\hline $\mathrm{M}_{3} \mathrm{Apt}_{1}$ & $\begin{array}{l}\text { GTTGAAGCAATACTCCCCCAGGTGCCACTTCACCTGGGGGAGTATTGCGG } \\
\text { AGGAAGGT }\end{array}$ \\
\hline $\mathrm{M}_{4} \mathrm{Apt}_{2}$ & $\begin{array}{l}\text { ATCTAACTGCTGCGCCGCCGGGAAAATACTGTACGGTTAGACACCTGGGG } \\
\text { GAGTATTGCTTCAACGCAATACTCCCCCAGGTGAAGTGG }\end{array}$ \\
\hline $\mathrm{M}_{4} \mathrm{C}$ & CACCTGGGGGAGTATTGCTTCAACGCAATACTCCCCCAGGTGAAGTGG \\
\hline Cy3-labeled $\mathrm{M}_{4} \mathrm{C}$ & $\begin{array}{l}\text { 5'-Су3- } \\
\text { CACCTGGGGGAGTATTGCTTCAACGCAATACTCCCCCAGGTGAAGTGG }\end{array}$ \\
\hline $\mathrm{cM}_{1} \mathrm{Apt}_{1}$ & $\begin{array}{l}\text { CCTTTAGCAATACTCCCCCAGGTGCCAAATCACCTGGGGGAGTATTGCAA } \\
\text { AAAAGAAT }\end{array}$ \\
\hline $\mathrm{cM}_{3} \mathrm{Apt}_{1}$ & $\begin{array}{l}\text { GTTGAAGCAATACTCCCCCAGGTGCCACTTCACCTGGGGGAGTATTGCAA } \\
\text { AAAAGAAT }\end{array}$ \\
\hline
\end{tabular}

Supplementary Table 2. The diameter of nanoparticles

\begin{tabular}{cc}
\hline Materials & Diameter (nm) \\
\hline AuNPs & $19.7 \pm 3.7$ \\
$\mathrm{~L}_{4} \mathrm{~B}_{0}$ & $21.2 \pm 3.6$ \\
$\mathrm{~L}_{4} \mathrm{~B}_{2}$ & $21.4 \pm 2.8$ \\
$\mathrm{~L}_{4} \mathrm{~B}_{4}$ & $22.2 \pm 2.6$ \\
$\mathrm{~L}_{4} \mathrm{~B}_{8}$ & $23.3 \pm 3.1$ \\
$\mathrm{~L}_{4} \mathrm{~B}_{12}$ & $26.6 \pm 5.4$ \\
SNAgel + ATP & $22.2 \pm 4.5$ \\
\hline
\end{tabular}

Supplementary Table 3. The hydrodynamic diameter of nanoparticles

\begin{tabular}{cc}
\hline Materials & Hydrodynamic diameter $(\mathbf{n m})$ \\
\hline AuNPs & 21.0 \\
SNAgel & 43.3 \\
SNAgel + ATP & 29.7 \\
SNAgel/Dox & 55.9 \\
SNAgel/Dox + ATP & 31.7 \\
\hline
\end{tabular}

